\title{
The Authors Reply "A Novel Configuration of a Traditional Rapid Response Team Decreases Non-Intensive Care Unit Arrests and Overall Hospital Mortality"
}

\author{
Daniel Davis, MD¹, Steve Aguilar, MD²
}

${ }^{1}$ Department of Emergency Medicine, University of California-San Diego, San Diego, California; '2Department of Nursing Education, Development, Research, University of California-San Diego, San Diego, California

We appreciate very much Dr. Singh's interest and insight regarding our article, "A Novel Configuration of a Traditional Rapid Response Team Decreases Non-Intensive Care Unit Arrests and Overall Hospital Mortality." 1 Dr. Singh makes several critical points that are worth emphasis and additional commentary.

The importance of cultural change in the success of a rapid response team (RRT) program cannot be emphasized enough. The willingness of frontline staff to access an RRT is based on a belief in the potential benefit to the patient as well as a lack of concern about the repercussions of such an activation, whether these are from the primary physician team or the RRT members themselves. Both of these require institutional commitment-ideally from administrative and clinical leadership-as well as routine, direct feedback to providers as to the effectiveness of the program. Both of these have been addressed in our advanced resuscitation training (ART) program, which has replaced traditional life-support training and consolidates many efforts related to patient safety and preventable death. ${ }^{2}$ The ART program represents "adaptive" training, in which arrest prevention is emphasized for non-intensive care unit staff and the importance of institutional processes such as RRT is emphasized.

Our approach to RRT configuration reflects the resource constraints referenced by Dr. Singh. Although the ideal RRT would include critical-care nurses located physically outside the intensive care unit to allow regular assessment of at-risk patients, this would have required expenditures that were not available for the program. In our opinion, a reasonable alternative was to train charge nurses from non-intensive care units as RRT members. The role expectation for these charge nurses included twice-daily rounds, and their proximity to at-risk patients facilitated regular reassessments throughout each shift. In addition, the ART program allowed routine training for bedside nurses to emphasize code/RRT issues on an annual basis and underscores the importance of early recognition of patient safety and preventable death. The ART program actually reduced life-support expenditures and allowed implementation of both our RRT and institutional cardiac arrest resuscitation programs in a costeffective manner.

The last point made by Dr. Singh that we wish to address involves the balance between over- and underutilization of RRT resources. Our RRT-to-code ratios are relatively favorable, allowing the program to exist with efficient allocation of resources. This may be due, in part, to the approach to training with regard to recognition of deterioration. Most RRT programs appear to emphasize vital sign thresholds or use of scoring systems for activation, both of which rely upon single sets of vital signs. Instead, we focus on pattern recognition, emphasizing dynamic changes in vital signs and other clinical assessments and de-emphasizing absolute values. We believe that this helps develop clinical decision-making skills and improves both sensitivity and specificity with regard to RRT activation. Again, the adaptive nature of the ART program allows annual training to enhance these skills without additional expense to the institution.

We very much appreciate Dr. Singh's comments and urge other institutions to listen to his message carefully. There is no substitute for efforts spent in establishing just culture and creating an institution that supports its staff in addressing patient safety issues, ultimately reducing preventable deaths.

\section{References}

1. Davis DP, Aguilar SA, Graham PG, et al. A novel configuration of a traditional rapid response team decreases non-intensive care unit arrests and overall hospital mortality. J Hosp Med. 2015;10:352-357.

2. Davis DP, Graham PG, Husa RD, et al. A performance improvementbased resuscitation programme reduces arrest incidence and increases survival from in-hospital cardiac arrest. Resuscitation. 2015;92:63-69. 